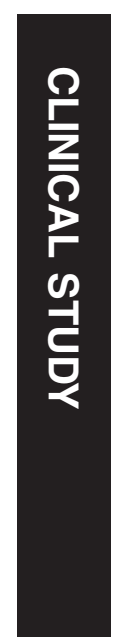

\section{A novel imaging approach to periocular basal cell carcinoma: in vivo optical coherence tomography and histological correlates}

${ }^{1}$ Ophthalmology Department, Maidstone \& Tunbridge Wells NHS

Trust, Maidstone, Kent, UK

${ }^{2}$ Histopathology

Department, Maidstone \&

Tunbridge Wells NHS

Trust, Maidstone, Kent, UK

${ }^{3}$ Michaelson Diagnostics

Ltd, 11A Grays Farm

Production Village,

Orpington, Kent, UK

Correspondence:

M Khandwala, Consultant Ophthalmic Surgeon,

Maidstone Hospital,

Hermitage Lane, ME16

9QQ, Maidstone, Kent, UK

Tel: +44 (0)1622 729 000;

Fax: +44 (0)1622 729000 .

E-mail:mona.khandwala@

gmail.com

Received: 23 November 2014

Accepted in revised form:

18 April 2015

Published online:

19 June 2015

Previous presentations: The results reported in this manuscript have been presented as a poster to Ever Meeting 2013 (Nice, France) and to World

Ophthalmology Congress 2014 (Tokyo, Japan)

\begin{abstract}
Purpose Optical coherence tomography (OCT) is a non-invasive imaging method widely used in ophthalmology. Recent developments have produced OCT devices for imaging the skin. The purpose of this study was to investigate Fourier Domain OCT morphological features of periocular basal cell carcinoma (BCC) in correlation with conventional histopathology.

Methods Consecutive patients with periocular nodular BCC were prospectively examined with VivoSight OCT (Michelson Ltd) prior to surgical excision. OCT slice mode images were analysed using criteria defined for conventional and HD-OCT; the images were correlated to haematoxylin and eosin stained histology sections.

Results A total of 15 patients with periocular BCC were recruited. Three categories of BCC morphological features were identified from slice mode OCT images: 1) Epidermal changes included epidermal thinning $(15 / 15 ; 100 \%)$, ulceration and crusting $(5 / 15,33.3 \%)$ and decreased density of hair follicles $(8 / 15 ; 53.3 \%)$; 2) Intralesional features included hyporeflective nodules $(15 / 15 ; 100 \%)$, hyper-reflective edges (15/15; $100 \%)$ and hyporeflective central necrosis $(3 / 15 ; 20 \%)$ ) Perilesional features included hyporeflective borders (11/15; 73\%), hypereflective margins $(15 / 15 ; 100 \%)$ and dilated blood vessels $(5 / 15 ; 33 \%)$.

Conclusions This study demonstrated that Fourier Domain OCT imaging offers additional information in the identification of morphological features of nodular BCC compared to conventional OCT diagnostic criteria. VivoSight produced fast, noninvasive imaging of skin lesions in the
\end{abstract}

L Pelosini ${ }^{1}$, HB Smith ${ }^{1}$, JB Schofield ${ }^{2}$ A Meeckings $^{3}$, A Dithal $^{1}$ and M Khandwala ${ }^{1}$ periocular region and high correlation with histology. Further studies are necessary to investigate OCT features of different histological subtypes of BCC. Eye (2015) 29, 1092-1098; doi:10.1038/eye.2015.97; published online 19 June 2015

\section{Introduction}

Basal cell carcinoma (BCC) is the commonest skin cancer in Caucasians over the age of 50 and accounts for 53000 new cases per year in the UK population. ${ }^{1,2}$ In the periocular region, BCCs represent $90 \%$ of all malignant tumors and occur most frequently in the lower eyelid (43\%), followed by the medial canthus (26\%), the upper eyelid $(12 \%)$, and the lateral canthus $(8 \%){ }^{1}$ Histologically, nodular and nodulocystic BCCs are the most common histological subtypes, representing $39.5 \%$ of all BCCs. ${ }^{3}$

The gold standard for diagnosis of BCC is represented by conventional surgical biopsy followed by histological evaluation. ${ }^{4}$ In view of the increasing incidence of BCC worldwide, non-invasive in vivo diagnostic techniques have been investigated, including reflectance confocal microscopy (RCM), 5,6 high frequency ultrasound (HFUS), 7,8 conventional optical coherence tomography (OCT $)^{9-13}$ and high-definition OCT (HD-OCT). ${ }^{14-16}$

Previous investigators demonstrated that RCM was a valuable tool in BCC diagnosis; however RCM required specific operator training and long contact time with the patient, achieving a penetration depth of approximately $250 \mu \mathrm{m}$, compared to $2 \mathrm{~mm}$ obtained with conventional OCT. ${ }^{5,6}$ HFUS was validated for the definition of BCC tumor thickness, although 
it was found to have insufficient specificity in the diagnosis of BCC. ${ }^{7,8}$

OCT was initially described in $1995^{17}$ for imaging of the retina and anterior chamber of the eye and was rapidly adopted in several medical fields due to its ease of use, non-invasiveness and short contact time with patients. OCT is an in vivo imaging technique based on the principle of Michelson interferometry and allows noninvasive, high-resolution, two or three-dimensional, cross-sectional imaging of tissues. The Michelson interferometer was invented by Albert Abraham Michelson and is best known for its use in the famous Michelson-Morley experiment (1887) which ruled out the existence of the aether. The device sends a light beam to the tissue to be analysed and records the signals generated by the interference of the light reflected from the tissue with a reference beam. The measurement of the interference pattern allows to determine the position of different tissue components and to provide contrast to the images. Albert Abraham Michelson (1852-1931) was a physicist and the first American to receive the Nobel Prize in Physics in 1907.

Welzel et $a l^{9,10}$ described the first OCT investigation of the human skin in 1997 and was followed by Olmedo et $a l^{11}$ and Mogensen et $a l^{12}$ who investigated OCT features of BCC, non-melanoma skin cancers (NMSC) and benign lesions versus normal skin. Conventional OCT studies for dermatological conditions demonstrated good visualisation of the classical layered microstructure of the skin and the ability to identify pathological features with more specificity than ultrasound. ${ }^{9-13}$ Time-Domain OCT systems however lacked both the contrast and penetration necessary for dermatological oncology.

Recently, HD-OCT devices have been developed, offering two major advantages over conventional OCT: an axial and lateral resolution of $3 \mu$ in tissue and the possibility of en-face imaging (coronal or C scan) as well as vertical slice imaging (B scan). ${ }^{14-16}$ Despite the higher resolution, the clinical applications of current HD-OCT devices are limited by a shallow penetration depth of $750 \mu$ and a small field of view of $1.8 \mathrm{~mm}$ by $1.5 \mathrm{~mm}$.

In this study, we investigated the Fourier-Domain 'VivoSight OCT' (Michelson Diagnostics Ltd, UK), a commercially available device, CE-marked (2010) and FDA-approved (2011) which features a multibeam system enabling a lateral resolution of $>7.5 \mu$ over a $1 \mathrm{~mm}$ focal range and a penetration depth of $1.2-1.8 \mathrm{~mm}$ in tissue.

There is limited evidence on the value of Fourier domain OCT for the identification of morphological correlates of BCC in comparison to histopathology. ${ }^{18}$ Whilst several authors have investigated conventional OCT and HD-OCT imaging for general dermatological use in various parts of the body, the OCT literature on periocular skin BCC is extremely limited. ${ }^{18,19}$
The purpose of this study was to investigate the correlation between histopathology features and Fourier Domain OCT features of periocular BCC in order to implement the current OCT diagnostic criteria for nodular BCC.

\section{Patients and methods}

This study was a prospective pilot OCT investigation for periocular BCC. The protocol of the study was approved by the institutional Research and Development Department of Maidstone and Tunbridge Wells NHS Trust and by the National Ethics Committee (Study Reference 12/NW/0478). This investigation was compliant with the Declaration of Helsinki and all participants signed a consent form before enrolment.

\section{Subjects, inclusion criteria and outcome measures}

Consecutive patients between 18 and 95 years of age with histologically proven periocular nodular BCC and willing to participate to all reviews were included in the study. Patients with previous history of eyelid surgery, recurrent $\mathrm{BCC}$ lesions and other periocular pathology were excluded from the study.

The main outcome measure of the study consisted of a qualitative analysis of OCT morphological features of periocular BCC in keeping with the OCT diagnostic criteria for BCC described by Olmedo et al ${ }^{11}$ and including: 1) dark lobular structure and pattern of abnormal architecture; 2) decreased reflectance at the periphery of the lobules; 3 ) highly reflective margins surrounding the lobules; 4) dilated blood vessels.

The secondary outcome measures were represented by OCT characteristics presenting in three different zones of the BCC tumour: 1) superficial epidermal features; 2) intralesional BCC features; 3) perilesional BCC features.

\section{Optical coherence tomography imaging}

OCT imaging of periocular BCC was performed using a commercially available Swept Source Fourier-Domain OCT, VivoSight OCT scanner (Michelson Diagnostics Ltd, UK). The system featured clinical CE mark (2010) and FDA approval for dermatology (2011), it conformed to laser safety Class 1 and was therefore eye-safe. This MultiBeam OCT system is capable of achieving between 1.2 and $1.8 \mathrm{~mm}$ imaging depth range, a $6 \times 6 \mathrm{~mm}$ lateral field of view and $<7.5 \mu \mathrm{m}$ lateral resolution and $<9 \mu \mathrm{m}$ vertical resolution in tissue. Each patient was examined with a standard raster scanning mode, recording 60 B-scans, $100 \mu \mathrm{m}$ apart and formed of pixels of $4 \mu$ in size.

OCT imaging was performed prior to surgical excision. During image acquisition, the probe was hand-held, 
located on the area of interest with the aid of a laser aiming beam (wavelength 645-664 nm) and maintained at the correct working-distance with a ring spacer $(6 \mathrm{~mm}$ in diameter). All scans were performed by the same investigator (LP) and no coupling gel was required for imaging. The quality of the image and the penetration of the OCT signal were assessed on the digital interface prior to image acquisition. For control reference purposes, the corresponding location of the patient's fellow eye was scanned before examining the BCC lesion.

B scan OCT images (vertical slice mode) were recorded with the same alignment as the histology cuts in order to achieve comparability of the sections. The orientation of the probe position with respect to the eyelid margin has been described in a previous publication. ${ }^{17}$

\section{Histopathology examination}

All BCC lesions were excised with predetermined $2 \mathrm{~mm}$ margins of normal skin and the four margins of each specimen were marked using $6 / 0$ silk sutures of different lengths. The excised specimens were transported to the histopathology Department in 10\% neutral buffered formalin, embedded in paraffin and stained with H\&E. Each specimen was cut into vertical sections similar to a 'bread-loaf' for histological examination and the report included the macroscopic, microscopic measurements, clear margins measurements and BCC description.

The BCC sections were examined by a histopathology consultant who was masked from the findings of the OCT scans, until routine histology reporting was completed. Once the analysis was completed, the OCT team and the histopathologist examined all sections in order to identify the best matching histologic section correlating with the OCT image for each of the 15 BCC. The detection rate of BCC features on OCT images was recorded and correlated with corresponding histological sections.

\section{Results}

\section{Patients and OCT imaging}

A total of 15 consecutive patients ( 3 females/12 males; age range: 49-91 years; mean age: 74 years) with periocular BCC underwent VivoSight OCT imaging. All 15 BCC lesions had a biopsy-proven diagnosis of nodular BCC located in the medial canthus $(6 / 15,40 \%)$, the lower lid $(5 / 15,33 \%)$, the lateral canthus $(3 / 15,20 \%)$ and the upper lid $(1 / 15,7 \%)$. The clinical data regarding patients and tumours characteristics are shown in Table 1.

The image acquisition with VivoSight SD-OCT was fast, painless and non-invasive. None of the patients reported discomfort or other adverse effects and the total contact time for imaging was less than $7 \mathrm{~min}$.

\section{Fourier domain OCT morphological features and histological correlates}

Fourier domain OCT slice images of BCC lesions and their histological correlates were analysed according to the diagnostic criteria described by Olmedo et al ${ }^{11}$ and morphological details in relation to the superficial features, intralesional features and perilesional features. Table 2 summarizes the detailed analysis of slice mode OCT scans.

Table 1 Patient and tumour characteristics: BCC histological measurements were recorded for the horizontal axis (X and $Y)$ and for the vertical axis $(Z$, depth of BCC)

\begin{tabular}{llccccc}
\hline ID & Gender & Age & Ethnicity & BCC Location & BCC histological type & Histology measurements $($ mm $) X \times Y \times Z$ \\
\hline 1 & M & 49 & White British & R, MC & Nodular & $8 \times 8 \times 0.7 \mathrm{~mm}$ \\
2 & M & 83 & Hispanic & L, UL & Nodular & $3 \times 4 \times 2.8$ \\
3 & M & 74 & White British & L, LL & Nodular & $9 \times 7.6 \times 0.7$ \\
4 & M & 79 & White British & R, MC & Nodular & $6.1 \times 4 \times 1$ \\
5 & M & 57 & White British & R, LL & Nodular & $6 \times 3.2 \times 1.6$ \\
6 & M & 65 & White British & R, LL & Nodular & $5 \times 5 \times 0.8$ \\
7 & M & 90 & White British & R, MC & Nodular & $6 \times 10 \times 1.5$ \\
8 & M & 82 & White British & L, LL & Nodular & $4 \times 4 \times 2$ \\
9 & F & 79 & White British & L, MC & Nodular & $4 \times 2.5 \times 1.2$ \\
10 & M & 87 & White British & R, MC & Nodular & $2.5 \times 4.7 \times 1$ \\
11 & M & 69 & White British & L, MC & Nodular & $5 \times 1.5 \times 1.5$ \\
12 & M & 91 & White British & R, LL & Nodular & $5 \times 5 \times 3$ \\
13 & M & 86 & White British & L, LL & Nodular & $3 \times 3 \times 1.5$ \\
14 & F & 55 & White British & R, LC & Nodular & $4 \times 4 \times 1.5$ \\
15 & F & 66 & White British & L, LC & Nodular & \\
\hline
\end{tabular}

Abbreviations: MC, medial canthus; UL, upper lid; LL, lower lid; LC, lateral canthus. The Y axis was also the axis of orientation of the OCT probe during imaging. 
Table 2 Detection rate of BCC morphological features with VivoSight Fourier Domain OCT

\begin{tabular}{|c|c|c|c|c|c|c|c|c|c|c|c|c|c|c|c|}
\hline \multirow[t]{2}{*}{ BCC Features } & \multicolumn{15}{|c|}{ Patients ID } \\
\hline & 1 & 2 & 3 & 4 & 5 & 6 & 7 & 8 & 9 & 10 & 11 & 12 & 13 & 14 & 15 \\
\hline \multicolumn{16}{|l|}{ Superficial epidermal features } \\
\hline Epidermal thinning 100\% (15/15) & + & + & + & + & + & + & + & + & + & + & + & + & + & + & + \\
\hline Crusting \& Ulceration $33.3 \%(5 / 15)$ & - & + & - & - & - & - & + & + & - & + & - & - & - & + & - \\
\hline Hair follicles $53.3 \%(8 / 15)$ & - & + & + & - & - & - & + & + & + & - & + & - & + & + & - \\
\hline \multicolumn{16}{|l|}{ Intralesional features } \\
\hline Hyporeflecive nodules $100 \%(15 / 15)$ & + & + & + & + & + & + & + & + & + & + & + & + & + & + & + \\
\hline Hypereflective edges $100 \%(15 / 15)$ & + & + & + & + & + & + & + & + & + & + & + & + & + & + & + \\
\hline Central necrosis $20 \%(3 / 15)$ & - & + & - & - & - & - & - & - & - & - & + & + & - & - & - \\
\hline \multicolumn{16}{|l|}{ Perilesional features } \\
\hline Hyporeflective borders 73\% (11/15) & + & - & + & + & + & + & - & + & + & - & + & + & + & + & - \\
\hline Hypereflective margins $100 \%(15 / 15)$ & + & + & + & + & + & + & + & + & + & + & + & + & + & + & + \\
\hline Dilated blood vessels 33.3\% (5/15) & + & + & - & - & + & - & - & + & - & - & + & - & - & - & - \\
\hline
\end{tabular}
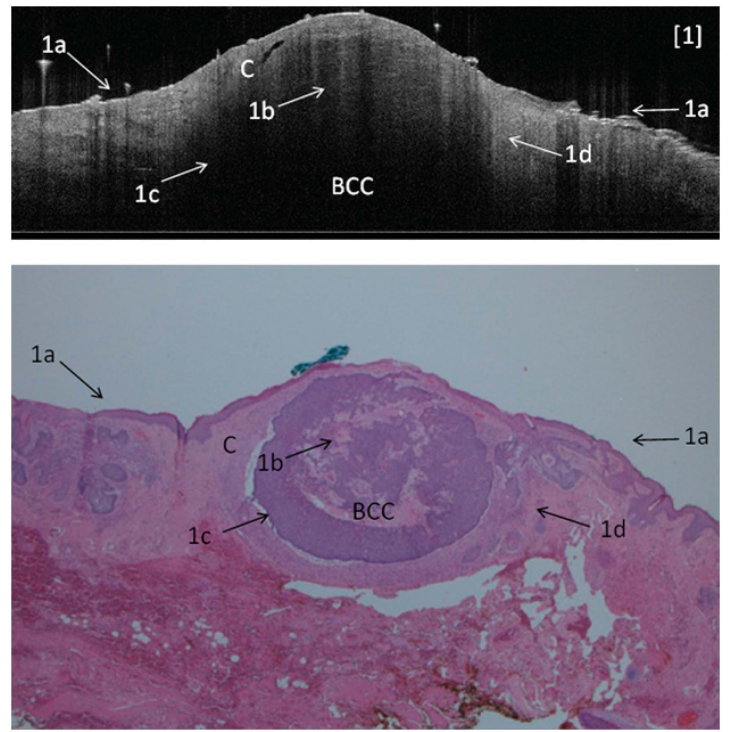

Figure 1 Example of nodular BCC with perilesional clefting (C) in Fourier OCT imaging and histology sections (H\&E stain, original magnification $\times 20)$. $1 \mathrm{a}=$ epidermal thinning and decreased density of hair follicles in proximity of BCC lesion; $1 \mathrm{~b}=$ intralesional low-reflectivity lobular pattern of BCC nests; $1 \mathrm{c}=$ perilesional dark border; $1 \mathrm{~d}=$ perilesional high-reflectivity margins of collagen compression.

\section{Superficial features}

Superficial features consisted in epidermal thinning $(15 / 15,100 \%)$, crusting and ulceration $(5 / 15,33 \%)$ and decreased density of hair follicles over the BCC nodule $(8 / 15,53 \%)$. The average pick-up rate of superficial features with VivoSight OCT was $62.2 \%$. The epidermis appeared as a high reflectivity signal on SD-OCT imaging
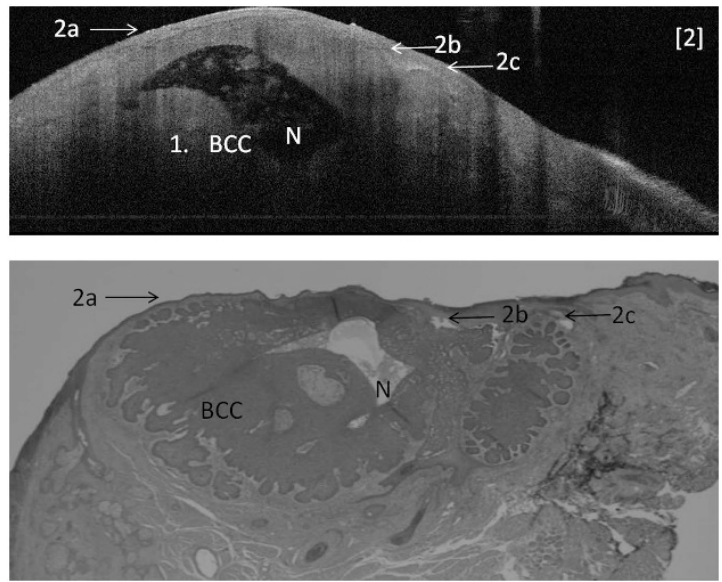

Figure 2 Example of large nodular BCC with central necrosis in Fourier domain OCT imaging and histology section (H\&E stain, original magnification $\times 20$ ). $2 a=$ epidermal thinning; $2 b=$ perilesional dark border of $\mathrm{BCC} ; 2 \mathrm{c}=$ perilesional high reflectivity margins; $\mathrm{N}=$ central necrosis; $\mathrm{BCC}=$ lobular pattern of lowreflectivity $\mathrm{BCC}$ nests.

(Figures 1 and 2). Superficial scabs and crusting appeared as high-reflectivity signal on OCT imaging, producing shadow cones and affecting the visualisation of the deeper structures. By contrast, ulceration generated a lowreflectivity signal. Hair follicles appeared as oblique tubular structures of low-reflectivity located in the superficial dermis surrounding the BCC lesion (Figure 1).

\section{Intralesional SD-OCT features}

Intralesional features consisted in hyporeflective nodules $(15 / 15,100 \%)$, hypereflective edges $(15 / 15,100 \%)$ and central necrosis $(3 / 15,20 \%)$. The average pick-up rate of intralesional features with VivoSight OCT was $73 \%$. 

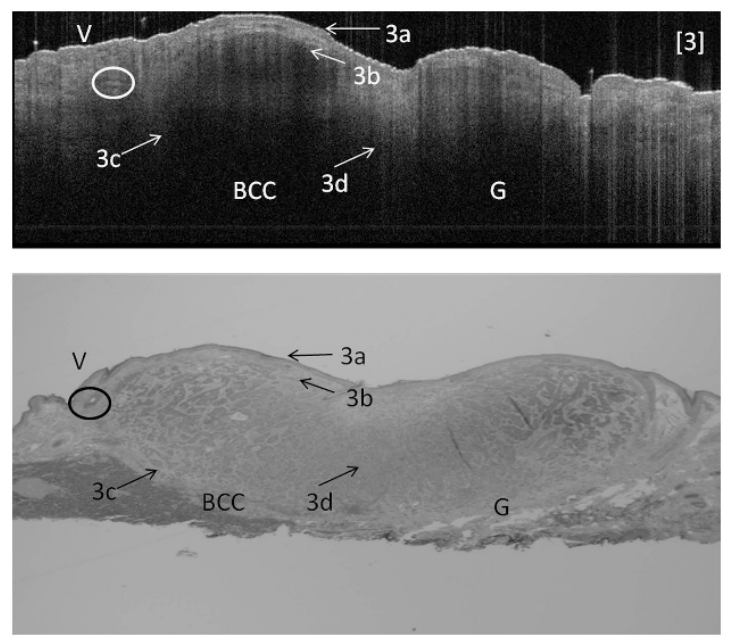

Figure 3 Example of nodular BCC in Fourier domain OCT imaging and histology section (H\&E stain, original magnification $\times 20$ ) showing 2 large lobular areas corresponding to two BCC nests (BCC, left) and a granuloma lesion ( $G$, right). $\mathrm{V}=$ perilesional dilated blood vessel in the upper dermis; $3 a=$ superficial epidermal thinning; $3 b=$ intralesional highreflectivity edge of $\mathrm{BCC}$ nest; $3 \mathrm{c}=$ perilesional low-reflectivity border; $3 \mathrm{~d}=$ high-reflectivity area of collagen fibres representing post-biopsy chronic inflammatory reaction.

All OCT images showed clusters of low-reflectivity lobules corresponding to high-cellularity BCC nests on the histology sections (Figure 1; Figure 3). High reflectivity OCT signal with linear pattern corresponded to belts of collagen compression secondary to progressive enlargement of adjacent BCC nests (Figure 3). Central necrosis appeared as low reflectivity cystic areas on OCT images containing highly reflective particles corresponding to proteinaceous aggregates on histology in the central part of the BCC (Figure 2).

\section{Perilesional SD-OCT features}

Perilesional features consisted in hyporeflective borders $(11 / 15,73 \%)$, hypereflective margins $(15 / 15,100 \%)$ and dilated blood vessels $(5 / 15,33 \%)$. The average pick-up rate of perilesional features with VivoSight OCT was $69 \%$. Low-reflectivity signal surrounding the tumour nodules was observed as dark crescents in OCT images and correlated with the location of clefts in corresponding histological sections (Figure 1). Hypereflective margins correlated with densely packed collagen fibres in the dermis correlating with perilesional chronic inflammation in the histology specimen (Figure 1; Figure 3). Dilated blood vessels were observed in the upper dermis and surrounding the tumour lesion (Figure 3).

\section{Discussion}

This prospective pilot study demonstrated the value of Fourier domain OCT imaging for the visualization of histopathological correlates of BCC.

Desirable features of in vivo imaging include noninvasiveness, high resolution, satisfactory tissue penetration, short patient contact time and ease of use. Fourier domain OCT imaging was painless, non-invasive and fast, the images were acquired without coupling gel, achieving $7.5 \mu$ resolution, $1.2-1.8 \mathrm{~mm}$ scanning depth and $6 \times 6 \mathrm{~mm}$ lateral field of view. By contrast, HD-OCT allows a higher resolution of $3 \mu$ in tissue, however the tissue penetration and the field of view of HD-OCT are limited to $750 \mu \mathrm{m}$ and $1.8 \times 1.5 \mathrm{~mm}$ respectively; the imaging probe requires a gel as coupling medium which could possibly cause side irritation in the periocular region. ${ }^{14-16}$

We classified the OCT characteristics of nodular BCC based on the anatomical distribution of the features with respect to the epidermis, the $\mathrm{BCC}$ lesion and the surrounding structures of the dermis.

Superficial epidermal changes have been commonly described as disarrangement of the epidermis in conventional OCT images and although they are not diagnostic features they acted as landmarks and provided localisation tracking during image acquisition. ${ }^{20,21}$

Fourier Domain OCT showed epidermal thinning, superficial crusting and rarefaction of the hair follicles; these features have not been described in HD-OCT reports. ${ }^{14-16}$ In keeping with our results, HD-OCT investigations have shown epidermal disarray in en-face scans and destruction of layering of the epidermis and dermis in slice mode scans with similar detection rates between the two imaging modes. ${ }^{14-16}$

The original OCT diagnostic criteria for BCC lesions were described by Olmedo et al using conventional OCT. ${ }^{11}$ In our study, Fourier domain imaging showed dark lobular patterns of BCC nests and bright margins of collagen compression in $100 \%$; decreased reflectance at the borders of the lobules was seen in $73 \%$ of the lesions, whereas dilated blood vessels were detected in only $33 \%$, in keeping with previous studies. ${ }^{15,18}$ The additional feature of central necrosis was observed in $20 \%$ of lesions and suggested the BCC growth pattern with fast progressive enlargement outgrowing the tissue blood supply.

Recent HD-OCT studies using Skyntell (Agfa) observed similar BCC diagnostic features in agreement with previous publications ${ }^{11,17,18}$ including dark lobulated nodules, peripheral rimming, peritumoral bright stroma and dilated blood vessels. Interestingly, the detection of lobulated nodules (84 vs $40 \%$ ), peritumoral bright stroma ( 88 vs $68 \%$ ) and dark peripheral rimming (72 vs 32\%) 
appeared to be consistently higher in HD-OCT en-face images compared to slice mode scans. ${ }^{16}$ Similarly, dilated blood vessels were detected in 50\% of en-face HD-OCT compared to $32 \%$ slice mode images. ${ }^{15}$ By contrast, the detection of superficial features such as epidermal disarray in en-face images and destruction of layers in slice mode shows similar detection rates. ${ }^{15,16}$ The higher sensitivity of en-face HD-OCT compared to slice mode could result from better resolution in superficial skin layers facilitating the detection of BCC features in horizontal sections with progressive signal degradation at increasing depth affecting the detection of features in slice mode.

The higher detection rate of BCC features in our study could be explained with the selectivity of this study for nodular BCC lesions and better penetration depth of Fourier domain OCT; the specific tumour subtype could possibly generate a higher incidence of specific features and the OCT penetration could facilitate the visualisation of lesional and perilesional features in slice mode at increasing tissue depths.

The dark peripheral border surrounding BCC nodules has been the subject of controversial interpretations; previous investigators suggested peritumoral oedema, palisading of cells, clefting and peritumoral mucin deposits. Although peritumoral oedema and clefting have always been interpreted as in vivo features with no histological correlates, clefting has been described in vivo with RCM, ${ }^{22}$ but never been described in OCT. ${ }^{15}$ Maier described the dark border as 'peripheral rimming' with higher detection rate in en face scan (77\%) compared to slice mode $(64 \%) .{ }^{15}$ Gambicher investigated the histological correlates of peripheral dark rimming using Alcian blue stain and confirmed the presence of mucin around BCC lobules. ${ }^{16}$ We identified histological clefting correlating with the dark border on Fourier OCT, suggesting the presence of this finding in vivo, as previously suggested by RCM investigations (Figure 1).

The strengths of our study included its prospective design, the comparison of histological sections and slice mode OCT with the same orientation and the masked analysis of the OCT and histopathology sections. Weaknesses of our study include the small number of patients, the investigation of only one BCC subtype and the lack of en-face OCT imaging. The study was designed as pilot investigation and the selectivity for nodular BCC was planned to achieve consistency of results in a small number of observations as nodular BCC is the most common type of BCC in clinical practice. En-face OCT is a valuable imaging modality, however it has several limitations: en-face is not directly comparable to histological sections, and does not allow BCC vertical margins measurement or localisation tracking during image acquisition.
In conclusion, VivoSight Fourier domain OCT appears to bridge the gap between conventional OCT imaging and HDOCT offering twice the lateral resolution of conventional single beam OCT systems (7.5 vs $15 \mu \mathrm{m}$ ) and twice the tissue penetration of HD-OCT $(1.2-1.8 \mathrm{~mm} v s 750 \mu \mathrm{m})$, therefore combining the best features of both systems.

The image acquisition with VivoSight required minimum contact with a limited area of the skin without the need of a coupling medium, making this technique particularly suitable for imaging BCC of the face and periocular region. This study has confirmed the potential of OCT as a non-invasive tool for the investigation of NMSC. Its role in dermatology has rapidly expanded over the last ten years in Germany, Switzerland and USA and its application to diagnose NMSC and premalignant conditions such as actinic keratosis in the periocular area is particularly important in order to reduce surgical excision. The use of OCT imaging could potentially replace invasive histological biopsies for NMSC and minimize removal of perilesional benign tissue. Improved imaging of BCCs is of great importance, not only for preoperative estimation of tumour thickness and margins, but also for identifying patients with superficial tumours suitable for non-invasive treatment strategies, such as 5-fluorouracil therapy. Furthermore, OCT imaging would allow the clinician to follow up the patient

postoperatively and assess full excision of the BCC and possible recurrence.

Further studies on Fourier domain OCT imaging are required to investigate morphological characteristics of different histological subtypes.

\section{Summary}

\section{What was known before}

- Conventional OCT can visualise the layered architecture of the skin with low resolution; By contrast, HD-OCT shows great details at cellular level with very shallow penetration in tissue. OCT imaging of the skin has been investigated extensively outside ophthalmology, however the literature on OCT imaging for the periocular skin is extremely limited.

What this study adds

- For the first time, Fourier OCT imaging allowed visualisation of periocular BCCs with high histopathological correlations of epidermal features, intralesional and perilesional characteristics Fourier domain OCT imaging offers higher resolution than conventional OCT and twice the tissue penetration of HDOCT.

\section{Conflict of interest}

The authors declare no conflict of interest. 


\section{References}

1 Cook Jr BE, Bartley GB. Epidemiologic characteristics and clinical course of patients with malignant eyelid tumours in an incidence cohort in Olmsted County, Minnesota. Ophthalmology 1999; 106: 746-750.

2 Margo CE, Waltz K. Basal cell carcinoma of the eyelid and periocular skin. Surv Ophthalmol 1993; 38: 169-192.

3 Malhotra R, Huilgol SC, Huyn NT, Selva D. The Australian Mohs Database, Part 2: periocular basal cell carcinoma outcome at 5 year follow up. Ophthalmology 2004; 111: 631-636.

4 Improving outcomes for people with skin tumours including melanoma (update): the management of low-risk basal cell carcinomas in the community. NICE Guidance on Cancer Services. May 2010.

5 Gonzalez S, Tannous Z. Real time, in vivo confocal reflectance microscopy of basal cell carcinoma. J Am Acad Dermatol 2002; 47: 869-874.

6 Nori S, Rius-Diaz F, Cuevas J, Goldgeier M, Jaen P, Torres A et al. Sensitivity and specificity of reflectance-mode confocal microscopy for in vivo diagnosis of basal cell carcinoma: a multicentre study. J Am Acad Dermatol 2004; 51: 923-930.

7 Mogensen M, Nurnberg B, Forman J, Thomsen JB, Thrane L, Jemec GB. In vivo thickness measurement of basal cell carcinoma and actinic keratosis with optical coherence tomography and 20-MHz ultrasound. Br J Dermatol 2009; 160: 1026-1033.

8 Hinz T, Ehler LK, Hornung T, Voth H, Fortmeier I, Maier T et al. Preoperative characterization of basal cell carcinoma comparing tumour thickness measurement by optical coherence tomography, 20-MHz ultrasound and histopathology. Acta Derm Venereol 2012; 92: 132-137.

9 Welzel J, Lankenau E, Birngruber R, Engelhardt R. Optical coherence tomography of the human skin. J Am Acad Dermatol 1997; 37(6): 958-963.

10 Welzel J. Optical coherence tomography in dermatology: a review. Skin Res Technol 2001; 7: 1-9.

11 Olmedo JM, Warschaw KE, Schmitt JM, Swanson DL. Optical coherence tomography for the characterization of basal cell carcinoma in vivo: a pilot study. J Am Acad Dermatol 2006; 55: 408-412.

12 Mogensen M, Joergensen TM, Nurnberg BM, Morsy HA, Thomsen JB, Thrane L et al. Assessment of optical coherence tomography imaging in the diagnosis of non-melanoma skin cancer and benign lesions versus normal skin: observer- blinded evaluation by dermatologists and pathologists. Dermatol Surg 2009; 35: 965-972.

13 Cunha D, Richardson T, Sheth N, Orchard G, Coleman A, Mallipeddi R. Comparison of ex vivo optical coherence tomography with conventional frozen-section histology for visualizing basal cell carcinoma during Mohs micrographic surgery. Br J Dermatol 2011; 165(3): 576-580.

14 Boone MA, Norrenberg S, Jemec GB, Del Marmol V. Imaging of basal cell carcinoma by high-definition optical coherence tomography: histomorphological correlation. A pilot study. Br J Dermatol 2012; 167: 856-864.

15 Maier T, Braun-Falco M, Hinz T, Schmid-Wendtner MH, Ruzicka T, Berking C. Morphology of basal cell carcinoma in high definition optical coherence tomography: en-face and slice imaging mode, and comparison with histology. J Eur Acad Dermatol Venereol 2013; 27(1): e97-104.

16 Gambichler T, Plura I, Kampilafkos P, Valavanis K, Sand M, Bechara FG et al. Histopathological correlates of basal cell carcinoma in slice and en-face imaging mode of high definition optical coherence tomography. Br J Dermatol 2014; 170(6): 1358-1361.

17 Fujimoto JG, Brezinski ME, Tearney GJ, Boppart SA, Bouma B, Hee MR et al. Optical biopsy and imaging using optical coherence tomography. Nat Med 1995; 1(9): 970-972.

18 Pelosini L, Smith HB, Schofield JB, Meeckings A, Dhital A, Khandwala M. In Vivo optical coherence tomography )OCT) in periocular basal cell carcinoma: correlations between in vivo OCT images and postoperative histology. $\mathrm{Br} J$ Ophthalmol 2013; 97(7): 890-894.

19 Khandwala M, Penmetsa BR, Dey S, Schofield JB, Jones CA, Podoleanu A. Imaging of periocular basal cell carcinoma using en face optical coherence tomography: a pilot study. Br J Ophthalmol 2010; 94: 1332-1336.

20 Mogensen M, Thrane L, Jorgensen TM, Andersen PE, Jemec GB. OCT imaging of skin cancer and other dermatological diseases. J Biophotonics 2009; 2: 442-451.

21 Jorgensen TM, Tycho A, Mogensen M, Bjerring P, Jemec GB. Machine-learning classification of non-melanoma skin cancers from image features obtained by optical coherence tomography. Skin Res Technol 2008; 14: 364-369.

22 Ulrich M, Roewert-Huber J, Gonzalez S, Rius-Diaz F, Stockfleth E, Kanitakis J. Peritumoral clefting in basal cell carcinoma: correlation of in vivo reflectance confocal microscopy and routine histology. J Cutan Pathol 2011; 38: 190-195. 